\title{
Article
}

\section{The Stability of Isometries on Restricted Domains}

\author{
Ginkyu Choi ${ }^{1,+}$ and Soon-Mo Jung ${ }^{2, *,+}$ \\ 1 Department of Electronic and Electrical Engineering, College of Science and Technology, Hongik University, \\ Sejong 30016, Korea; gkchoi@hongik.ac.kr \\ 2 Mathematics Section, College of Science and Technology, Hongik University, Sejong 30016, Korea \\ * Correspondence: smjung@hongik.ac.kr; Tel.: +82-44-860-2584 \\ + These authors contributed equally to this work.
}

check for

updates

Citation: Choi, G.; Jung, S.-M. The Stability of Isometries on Restricted Domains. Symmetry 2021, 13, 282. https://doi.org/10.3390/sym13020282

Academic Editor: Marin Marin, Włodzimierz Fechner, Jacek Chudziak Received: 22 December 2020 Accepted: 1 February 2021 Published: 7 February 2021

Publisher's Note: MDPI stays neutral with regard to jurisdictional clai$\mathrm{ms}$ in published maps and institutional affiliations.

Copyright: $@ 2021$ by the authors. Licensee MDPI, Basel, Switzerland. This article is an open access article distributed under the terms and conditions of the Creative Commons Attribution (CC BY) license (https:// creativecommons.org/licenses/by/ $4.0 /)$.

\begin{abstract}
We will prove the generalized Hyers-Ulam stability of isometries, with a focus on the stability for restricted domains. More precisely, we prove the generalized Hyers-Ulam stability of the orthogonality equation and we use this result to prove the stability of the equations $\|f(x)-f(y)\|=$ $\|x-y\|$ and $\|f(x)-f(y)\|^{2}=\|x-y\|^{2}$ on the restricted domains. As we can easily see, these functional equations are symmetric in the sense that they become the same equations even if the roles of variables $x$ and $y$ are exchanged.
\end{abstract}

Keywords: isometry; linear isometry; Hyers-Ulam stability; generalized Hyers-Ulam stability; restricted domain

\section{Introduction}

Throughout this paper, unless otherwise mentioned, $(E,\|\cdot\|)$ and $(F,\|\cdot\|)$ are used to denote Hilbert spaces over $\mathbb{K}$, where $\mathbb{K}$ is either $\mathbb{R}$ or $\mathbb{C}$. A mapping $f: E \rightarrow F$ is said to be an isometry if $f$ satisfies:

$$
\|f(x)-f(y)\|=\|x-y\|
$$

for any $x, y \in E$.

By considering the definition of Hyers and Ulam [1], for each fixed $\varepsilon \geq 0$, a function $f: E \rightarrow F$ is said to be an $\varepsilon$-isometry if $f$ satisfies the inequality:

$$
|\|f(x)-f(y)\|-\|x-y\|| \leq \varepsilon
$$

for any $x, y \in E$. If there exists a positive constant $K$ such that for each $\varepsilon$-isometry $f: E \rightarrow F$, there is an isometry $U: E \rightarrow F$ satisfying the inequality $\|f(x)-U(x)\| \leq K \varepsilon$ for every $x \in E$, then the functional Equation (1) is said to have (or satisfy) the Hyers-Ulam stability.

We can weaken the conditions associated with Hyers-Ulam stability in order to study more diverse topics as follows: Assume that $\varphi, \Phi: E \times E \rightarrow[0, \infty)$ satisfy some mild conditions. If for each function $f: E \rightarrow F$ that satisfies the inequality:

$$
|\|f(x)-f(y)\|-\|x-y\|| \leq \varphi(x, y),
$$

there exists a function $U: E \rightarrow F$ such that $\|U(x)-U(y)\|=\|x-y\|$ for any $x, y \in E$ and $\|f(x)-U(x)\| \leq \Phi(x, x)$ for any $x \in E$, then the functional Equation (1) is said to have (or satisfy) the generalized Hyers-Ulam stability. We use these terminologies also for other types of functional equations.

To the best of our knowledge, Hyers and Ulam were the first mathematicians to study the Hyers-Ulam stability of isometries (see [1]). Indeed, they were able to prove the stability of isometries based on the properties of the inner product of Hilbert spaces: For each surjective $\varepsilon$-isometry $f: E \rightarrow E$ satisfying $f(0)=0$, there is a surjective isometry $U: E \rightarrow E$ satisfying $\|f(x)-U(x)\| \leq 10 \varepsilon$ for every $x \in E$. 
The theorem of Hyers and Ulam was further generalized by Bourgin [2]: Assume that $E$ is a real Banach space and that $F$ belongs to the class of uniformly convex real Banach spaces which includes the spaces $L_{p}(0,1)$ for $1<p<\infty$. For any $\varepsilon$-isometry $f: E \rightarrow F$ with $f(0)=0$, there exists a linear isometry $U: E \rightarrow F$ such that $\|f(x)-U(x)\| \leq 12 \varepsilon$ for every $x \in E$.

Thereafter, Hyers and Ulam [3] investigated the stability problem for the Banach spaces of continuous functions: For each $i \in\{1,2\}$, let $S_{i}$ be a compact metric space and let $C\left(S_{i}\right)$ be the Banach space of real-valued continuous functions on $S_{i}$ equipped with the supremum norm $\|\cdot\|_{\infty}$. If a homeomorphism $T: C\left(S_{1}\right) \rightarrow C\left(S_{2}\right)$ satisfies the inequality:

$$
\left|\|T(f)-T(g)\|_{\infty}-\|f-g\|_{\infty}\right| \leq \varepsilon
$$

for some $\varepsilon>0$ and for any $f, g \in C\left(S_{1}\right)$, then there exists an isometry $U: C\left(S_{1}\right) \rightarrow C\left(S_{2}\right)$ satisfying $\|T(f)-U(f)\|_{\infty} \leq 21 \varepsilon$ for any $f \in C\left(S_{1}\right)$.

The very result of Hyers and Ulam was profoundly generalized once again by Bourgin [4]: If $S_{i}$ is a completely regular Hausdorff space for $i \in\{1,2\}$ and $T: C\left(S_{1}\right) \rightarrow C\left(S_{2}\right)$ is a surjective function satisfying the inequality (3) for some $\varepsilon>0$ and for all $f, g \in C\left(S_{1}\right)$, then there exists a linear isometry $U: C\left(S_{1}\right) \rightarrow C\left(S_{2}\right)$ satisfying $\|T(f)-U(f)\|_{\infty} \leq 10 \varepsilon$ for any $f \in C\left(S_{1}\right)$. The study of the stability of isometries was then continued by Bourgin for the finite-dimensional Banach spaces (see [5]).

In 1978, Gruber [6] proved the following theorem: Assume that $E$ and $F$ are real normed spaces, $f: E \rightarrow F$ is a surjective $\varepsilon$-isometry, and that $U: E \rightarrow F$ is an isometry satisfying $f(p)=U(p)$ for a $p \in E$. If $\|f(x)-U(x)\|=o(\|x\|)$ as $\|x\| \rightarrow \infty$ uniformly, then $U$ is a surjective linear isometry and $\|f(x)-U(x)\| \leq 5 \varepsilon$ for all $x \in E$. In particular, if $f$ is continuous, then $\|f(x)-U(x)\| \leq 3 \varepsilon$ for each $x \in E$.

Years later, Gevirtz [7] proved the stability of isometries defined between real Banach spaces: Assume that $E$ and $F$ are real Banach spaces. For each surjective $\varepsilon$-isometry $f: E \rightarrow F$, there exists a surjective isometry $U: E \rightarrow F$ such that $\|f(x)-U(x)\| \leq 5 \varepsilon$ for any $x \in E$. After that, the upper bound $5 \varepsilon$ was improved to a sharper $2 \varepsilon$ by Omladič and Šemrl [8].

According to [9,10], a function $f: E \rightarrow F$ is called an $(\varepsilon, p)$-isometry when $f$ satisfies:

$$
|\|f(x)-f(y)\|-\|x-y\|| \leq \varepsilon\|x-y\|^{p}
$$

for some constants $p \geq 0$ and $\varepsilon \geq 0$ and for all $x, y \in E$. Dolinar [9] proved the superstability of $(\varepsilon, p)$-isometries: When $p>1$, every surjective $(\varepsilon, p)$-isometry $f: E \rightarrow F$ between finite-dimensional real Banach spaces is an isometry.

There are many other papers related to the stability of isometries, but it is regrettable that due to the restriction of space, they cannot be quoted one by one. Nevertheless, see [11-25] for more general information on the stability of isometries and related topics.

The following theorem of Fickett provided an important motivation for writing this paper. Fickett [15] proved the following theorem:

Theorem 1. For a fixed integer $n \geq 2$, let $S$ be a convex bounded subset of $\mathbb{R}^{n}$ and let $\varepsilon>0$ be given. If a function $f: S \rightarrow \mathbb{R}^{n}$ satisfies the inequality:

$$
|\|f(x)-f(y)\|-\|x-y\|| \leq \varepsilon
$$

for all $x, y \in S$, then there exists an isometry $U: S \rightarrow \mathbb{R}^{n}$ such that:

$$
\|f(x)-U(x)\| \leq 27 \varepsilon^{1 / 2^{n}}
$$

for each $x \in S$. 
The upper bound associated with inequality in Fickett's theorem becomes very large for any sufficiently small $\varepsilon$ in comparison to $\varepsilon$. This is a big drawback of Fickett's theorem. Thus, the work of further improving Fickett's theorem has to be attractive.

In this paper, we prove the generalized Hyers-Ulam stability of isometries with a focus on the stability for restricted domains. More precisely, we prove the generalized HyersUlam stability of the orthogonality equation and we apply this result for proving some theorems about the generalized Hyers-Ulam stability of equations $\|f(x)-f(y)\|=\|x-y\|$ and $\|f(x)-f(y)\|^{2}=\|x-y\|^{2}$, which are equivalent to each other in the sense that their solution sets are the same. As can be easily seen, those functional equations are symmetric in that they become the same equations even if the variables $x$ and $y$ are exchanged. For readers interested in the stability and related topics of the orthogonality equation, read the papers $[13,14,26-29]$.

\section{Preliminaries}

In this section, $E$ and $F$ denote the Hilbert spaces over $\mathbb{K}$, where $\mathbb{K}$ denotes either $\mathbb{R}$ or $\mathbb{C}$. We note that a function $f: E \rightarrow F$ satisfies the orthogonality equation:

$$
\langle f(x), f(y)\rangle=\langle x, y\rangle
$$

for all $x, y \in E$ if and only if it is a linear isometry.

A function $f: E \rightarrow F$ is called conjugate-linear if and only if $f(\lambda x+\mu y)=\bar{\lambda} f(x)+$ $\bar{\mu} f(y)$ for all $x, y \in E$ and $\lambda, \mu \in \mathbb{K}$. We note that a function $f: E \rightarrow F$ satisfies the equation:

$$
\langle f(x), f(y)\rangle=\langle y, x\rangle
$$

for any $x, y \in E$ if and only if it is a conjugate-linear isometry.

Let $\mathcal{S}=\{z \in \mathbb{K}:|z|=1\}$. Every two functions $f, g: E \rightarrow F$ are called phase-equivalent if and only if there is a function $\sigma: E \rightarrow \mathcal{S}$ satisfying $g(x)=\sigma(x) f(x)$ for all $x \in E$.

The following equation:

$$
|\langle f(x), f(y)\rangle|=|\langle x, y\rangle|
$$

is known as the Wigner equation or the generalized orthogonality equation. Wigner introduced the following theorem in [30].

Theorem 2. If a function $f: E \rightarrow F$ satisfies the Wigner Equation (5) for all $x, y \in E$, then $f$ is phase-equivalent to a linear isometry or a conjugate-linear isometry.

Let $(E,\langle\cdot, \cdot\rangle)$ be a Hilbert space with the associated norm defined as $\|x\|=\sqrt{|\langle x, x\rangle|}$. For any fixed $d \geq 0$, we define either $D=\{x \in E:\|x\| \geq d\}$ or $D=\{x \in E:\|x\| \leq d\}$. We now select a constant $c$ satisfying the condition:

$$
\begin{cases}0<c<1 & (\text { for } D=\{x \in E:\|x\| \geq d\}), \\ c>1 & (\text { for } D=\{x \in E:\|x\| \leq d\}) .\end{cases}
$$

In this paper, we will exclude the trivial case $D=\{0\}$. In addition, let us define $D_{0}=D \cup\{0\}$. We note that we select the constant $c$ and domain $D$ with the definition (6) in mind.

The following theorem has been proved in Theorem 2 of [26] which deals with the generalized Hyers-Ulam stability of the orthogonality Equation (4).

Theorem 3. Assume that a function $\varphi: E \times E \rightarrow[0, \infty)$ satisfies the condition:

$$
\lim _{m+n \rightarrow \infty} c^{m+n} \varphi\left(\frac{1}{c^{m}} x, \frac{1}{c^{n}} y\right)=0
$$


for any $x, y \in D$. If a function $f: E \rightarrow F$ satisfies the inequality:

$$
|\langle f(x), f(y)\rangle-\langle x, y\rangle| \leq \varphi(x, y)
$$

for all $x, y \in D$, then there exists a unique linear isometry $U: E \rightarrow F$ satisfying:

$$
\|f(x)-U(x)\| \leq \sqrt{\varphi(x, x)}
$$

for any $x \in D$.

A normed space $(E,\|\cdot\|)$ is called strictly convex if and only if for any given $x, y \in E$, $\|x+y\|=\|x\|+\|y\|$ implies that $x$ and $y$ are linearly dependent.

Baker demonstrated the following theorem in [11].

Theorem 4. If $E$ is a normed space over $\mathbb{R}$ and $F$ is a strictly convex normed space over $\mathbb{R}$, then every isometry $f: E \rightarrow F$ is affine. In particular, a function $f: E \rightarrow F$ is an isometry and $f(0)=0$ if and only if $f$ is a linear isometry.

\section{Main Result}

The condition of the following theorem is weaker than condition (7) of Theorem 3. Indeed, the new condition (8) does not look much different from the old one (7), but we will see from theorems and corollaries in the following section that there are quite significant differences in their utilizations. That is, Theorem 5 is an improved version of Theorem 3 and it will play a central role in this paper.

The proof of the following theorem is based strongly on the well known properties of the inner product of Hilbert spaces and the direct method that was first conceived by Hyers [31].

Theorem 5. Let $E$ and $F$ be Hilbert spaces over $\mathbb{K}$ and assume that a function $\varphi: E \times E \rightarrow[0, \infty)$ satisfies the condition:

$$
\lim _{m, n \rightarrow \infty} c^{m+n} \varphi\left(\frac{1}{c^{m}} x, \frac{1}{c^{n}} y\right)=0
$$

for all $x, y \in D$. If a function $f: E \rightarrow F$ satisfies the inequality:

$$
|\langle f(x), f(y)\rangle-\langle x, y\rangle| \leq \varphi(x, y)
$$

for all $x, y \in D$, then there exists a unique linear isometry $U: E \rightarrow F$ such that:

$$
\|f(x)-U(x)\| \leq \sqrt{\varphi(x, x)+\lim _{n \rightarrow \infty} c^{n} \varphi\left(\frac{1}{c^{n}} x, x\right)+\lim _{n \rightarrow \infty} c^{n} \varphi\left(x, \frac{1}{c^{n}} x\right)}
$$

for all $x \in D$. 
Proof. We select a constant $c$ satisfying the condition (6). By the definition of norm and the triangle inequality, ||$a|-| b|| \leq|a-b|$ for all $a, b \in \mathbb{C}$, and since $c^{n} \frac{1}{c^{n}} x-c^{m} \frac{1}{c^{m}} x=0$ and $\|w\|^{2}=\langle w, w\rangle$ for all $w \in F$, we have:

$$
\begin{aligned}
& \left\|c^{n} f\left(\frac{1}{c^{n}} x\right)-c^{m} f\left(\frac{1}{c^{m}} x\right)\right\|^{2} \\
& =\left|\left\|c^{n} f\left(\frac{1}{c^{n}} x\right)-c^{m} f\left(\frac{1}{c^{m}} x\right)\right\|^{2}-\left\|c^{n} \frac{1}{c^{n}} x-c^{m} \frac{1}{c^{m}} x\right\|^{2}\right| \\
& =\|\left\langle c^{n} f\left(\frac{1}{c^{n}} x\right)-c^{m} f\left(\frac{1}{c^{m}} x\right), c^{n} f\left(\frac{1}{c^{n}} x\right)-c^{m} f\left(\frac{1}{c^{m}} x\right)\right\rangle \mid \\
& \quad-\left|\left\langle c^{n} \frac{1}{c^{n}} x-c^{m} \frac{1}{c^{m}} x, c^{n} \frac{1}{c^{n}} x-c^{m} \frac{1}{c^{m}} x\right\rangle\right| \mid \\
& \leq \mid\left\langle c^{n} f\left(\frac{1}{c^{n}} x\right)-c^{m} f\left(\frac{1}{c^{m}} x\right), c^{n} f\left(\frac{1}{c^{n}} x\right)-c^{m} f\left(\frac{1}{c^{m}} x\right)\right\rangle \\
& \quad-\left\langle c^{n} \frac{1}{c^{n}} x-c^{m} \frac{1}{c^{m}} x, c^{n} \frac{1}{c^{n}} x-c^{m} \frac{1}{c^{m}} x\right\rangle \mid
\end{aligned}
$$

for any $x \in D$ and $m, n \in \mathbb{N}_{0}$. We note that $\frac{1}{c^{m}} x \in D$ and $\frac{1}{c^{n}} x \in D$ for each $x \in D$ and any $m, n \in \mathbb{N}_{0}$. Hence, by using (9), the last inequality and triangle inequality, we get:

$$
\begin{aligned}
\left\|c^{n} f\left(\frac{1}{c^{n}} x\right)-c^{m} f\left(\frac{1}{c^{m}} x\right)\right\|^{2} \\
\leq\left|\left\langle c^{n} f\left(\frac{1}{c^{n}} x\right), c^{n} f\left(\frac{1}{c^{n}} x\right)\right\rangle-\left\langle c^{n} \frac{1}{c^{n}} x, c^{n} \frac{1}{c^{n}} x\right\rangle\right| \\
\quad+\left|\left\langle c^{n} f\left(\frac{1}{c^{n}} x\right), c^{m} f\left(\frac{1}{c^{m}} x\right)\right\rangle-\left\langle c^{n} \frac{1}{c^{n}} x, c^{m} \frac{1}{c^{m}} x\right\rangle\right| \\
\quad+\left|\left\langle c^{m} f\left(\frac{1}{c^{m}} x\right), c^{n} f\left(\frac{1}{c^{n}} x\right)\right\rangle-\left\langle c^{m} \frac{1}{c^{m}} x, c^{n} \frac{1}{c^{n}} x\right\rangle\right| \\
\quad+\left|\left\langle c^{m} f\left(\frac{1}{c^{m}} x\right), c^{m} f\left(\frac{1}{c^{m}} x\right)\right\rangle-\left\langle c^{m} \frac{1}{c^{m}} x, c^{m} \frac{1}{c^{m}} x\right\rangle\right| \\
\leq c^{2 n} \varphi\left(\frac{1}{c^{n}} x, \frac{1}{c^{n}} x\right)+c^{n+m} \varphi\left(\frac{1}{c^{n}} x, \frac{1}{c^{m}} x\right)+c^{m+n} \varphi\left(\frac{1}{c^{m}} x, \frac{1}{c^{n}} x\right)+c^{2 m} \varphi\left(\frac{1}{c^{m}} x, \frac{1}{c^{m}} x\right)
\end{aligned}
$$

for all $x \in D$ and $m, n \in \mathbb{N}_{0}$.

On account of (8) and (11), we see that for each $x \in D$, the sequence $\left\{c^{n} f\left(\frac{1}{c^{n}} x\right)\right\}$ is a Cauchy sequence in a Hilbert space $F$. Hence, we can define a function $u: D \rightarrow F$ by:

$$
u(x)=\lim _{n \rightarrow \infty} c^{n} f\left(\frac{1}{c^{n}} x\right)
$$

for every $x \in D$.

We will now extend the domain of $u$ from $D$ to the whole space $E$. For any $x \in E \backslash\{0\}$, we set:

$$
n(x)=\min \left\{n \in \mathbb{N}_{0}: \frac{1}{c^{n}} x \in D\right\}
$$

and we know that $c^{-n(x)} x \in D$ for any $x \in E \backslash\{0\}$. Thus, we can define a function $U: E \rightarrow F$ by:

$$
U(x)= \begin{cases}c^{n(x)} u\left(c^{-n(x)} x\right) & (\text { for } x \neq 0) \\ 0 & (\text { for } x=0)\end{cases}
$$


For $x=0$ or $y=0$, it is obvious that $\langle U(x), U(y)\rangle=0=\langle x, y\rangle$ because $U(x)=x=0$ or $U(y)=y=0$. When $x, y \in E \backslash\{0\}$, we note that $\frac{1}{c^{n(x)}} x \in D, \frac{1}{c^{n(y)}} y \in D, \frac{1}{c^{n+n(x)}} x \in D$, and $\frac{1}{c^{m+n(y)}} y \in D$ for any $m, n \in \mathbb{N}_{0}$, and it follows from (8) and (9) that:

$$
\begin{aligned}
&|\langle U(x), U(y)\rangle-\langle x, y\rangle| \\
&=\left|\left\langle c^{n(x)} u\left(\frac{1}{c^{n(x)}} x\right), c^{n(y)} u\left(\frac{1}{c^{n(y)}} y\right)\right\rangle-c^{n(x)+n(y)}\left\langle\frac{1}{c^{n(x)}} x, \frac{1}{c^{n(y)}} y\right\rangle\right| \\
&=\mid\left\langle c^{n(x)} \lim _{n \rightarrow \infty} c^{n} f\left(\frac{1}{c^{n+n(x)}} x\right), c^{n(y)} \lim _{m \rightarrow \infty} c^{m} f\left(\frac{1}{c^{m+n(y)}} y\right)\right\rangle \\
&-c^{n(x)+n(y)}\left\langle\frac{1}{c^{n(x)}} x, \frac{1}{c^{n(y)}} y\right\rangle \mid \\
&= c^{n(x)+n(y)}\left|\left\langle\lim _{n \rightarrow \infty} c^{n} f\left(\frac{1}{c^{n+n(x)}} x\right), \lim _{m \rightarrow \infty} c^{m} f\left(\frac{1}{c^{m+n(y)}} y\right)\right\rangle-\left\langle\frac{1}{c^{n(x)}} x, \frac{1}{c^{n(y)}} y\right\rangle\right| \\
&= \lim _{m, n \rightarrow \infty} c^{n(x)+n(y)+n+m}\left|\left\langle f\left(\frac{1}{c^{n+n(x)}} x\right), f\left(\frac{1}{c^{m+n(y)}} y\right)\right\rangle-\left\langle\frac{1}{c^{n+n(x)}} x, \frac{1}{c^{m+n(y)}} y\right\rangle\right| \\
& \leq c^{n(x)+n(y)} \lim _{m, n \rightarrow \infty} c^{n+m} \varphi\left(\frac{1}{c^{n}} \frac{1}{c^{n(x)}} x, \frac{1}{c^{m}} \frac{1}{c^{n(y)}} y\right) \\
&= 0 .
\end{aligned}
$$

That is, $U$ is a solution to the orthogonality equation. In other words, $U$ is a linear isometry.

If we set $m=0$ and let $n$ go to infinity in (11), then we get the inequality (10). It now remains to prove the uniqueness of $U$. Let $V: E \rightarrow F$ be another linear isometry satisfying the inequality (10) for all $x \in D$. By the linearity of $U$ and $V$, we know by using (8) that:

$$
\begin{aligned}
& \|U(x)-V(x)\| \\
& =\left\|c^{m} U\left(\frac{1}{c^{m}} x\right)-c^{m} f\left(\frac{1}{c^{m}} x\right)\right\|+\left\|c^{m} f\left(\frac{1}{c^{m}} x\right)-c^{m} V\left(\frac{1}{c^{m}} x\right)\right\| \\
& \leq 2 c^{m} \sqrt{\varphi\left(\frac{1}{c^{m}} x, \frac{1}{c^{m}} x\right)+\lim _{n \rightarrow \infty} c^{n} \varphi\left(\frac{1}{c^{m+n}} x, \frac{1}{c^{m}} x\right)+\lim _{n \rightarrow \infty} c^{n} \varphi\left(\frac{1}{c^{m}} x, \frac{1}{c^{m+n}} x\right)} \\
& \rightarrow 0 \text { as } m \rightarrow \infty,
\end{aligned}
$$

for all $x \in D$. By using the linearity of $U$ and $V$ again, we can conclude that $U(x)=V(x)$ for all $x \in E$.

As we can see in the corollaries of the following section, in many application cases of Theorem 5, the limit values in the inequality (10) become zero.

\section{Stability of Isometries}

Assume temporarily that $f: E \rightarrow F$ is a function satisfying $f(0)=0$, where $E$ is a real Hilbert space and $F$ is a strictly convex real Hilbert space. In view of Theorem 4 with $f(0)=0, f$ is an isometry if and only if $f$ is a linear isometry. Therefore, when $E$ is a real Hilbert space and $F$ is a strictly convex real Hilbert space, the functional Equation (1) is substantially the same as the orthogonality Equation (4).

The stability problem for the functional Equation (1) will be addressed in the following theorem, which is somewhat closely related to the theorems demonstrated in $[16,17,32]$. We recall that we select a constant $c$ satisfying the condition (6), i.e.,

$$
\begin{cases}0<c<1 & (\text { for } D=\{x \in E:\|x\| \geq d\}), \\ c>1 & (\text { for } D=\{x \in E:\|x\| \leq d\})\end{cases}
$$

and $D_{0}=D \cup\{0\}$, where $d$ is an arbitrarily given nonnegative constant. 
Theorem 6. Let $E$ and $F$ be Hilbert spaces over $\mathbb{R}$. Assume that a function $\varphi: E \times E \rightarrow[0, \infty)$ satisfies the following conditions:

$$
\lim _{m, n \rightarrow \infty} c^{m+n}\left\|\frac{1}{c^{m}} x-\frac{1}{c^{n}} y\right\| \varphi\left(\frac{1}{c^{m}} x, \frac{1}{c^{n}} y\right)=0
$$

and

$$
\lim _{m, n \rightarrow \infty} c^{m+n} \varphi\left(\frac{1}{c^{m}} x, \frac{1}{c^{n}} y\right)^{2}=0
$$

for all $x, y \in D_{0}$. If a function $f: E \rightarrow F$ satisfies the inequality:

$$
|\|f(x)-f(y)\|-\|x-y\|| \leq \varphi(x, y)
$$

for all $x, y \in D_{0}$, then there exists a unique linear isometry $U: E \rightarrow F$ such that:

$$
\|f(x)-U(x)-f(0)\| \leq \sqrt{\psi(x, x)+\lim _{n \rightarrow \infty} c^{n} \psi\left(\frac{1}{c^{n}} x, x\right)+\lim _{n \rightarrow \infty} c^{n} \psi\left(x, \frac{1}{c^{n}} x\right)}
$$

for all $x \in D_{0}$, where:

$\psi(x, y)=\|x-y\| \varphi(x, y)+\|x\| \varphi(x, 0)+\|y\| \varphi(0, y)+\frac{1}{2} \varphi(x, y)^{2}+\frac{1}{2} \varphi(x, 0)^{2}+\frac{1}{2} \varphi(0, y)^{2}$

for any $x, y \in E$.

Proof. If we put $g(x)=f(x)-f(0)$ in (12), then we see that $g$ also satisfies the inequality (12) and $g(0)=0$. It follows from (12) with $g$ in place of $f$ that:

$$
\|g(x)-g(y)\|+\|x-y\| \leq 2\|x-y\|+\varphi(x, y)
$$

for any $x, y \in D_{0}$, since $\|g(x)-g(y)\| \leq\|x-y\|+\varphi(x, y)$ by (12).

Obviously, it holds that:

$$
\begin{aligned}
\langle g(x), g(y)\rangle-\langle x, y\rangle & \\
= & \langle g(x)-g(y), g(y)-g(x)\rangle+\langle g(x)-g(y), g(x)\rangle+\langle g(y), g(y)\rangle \\
& \quad-\langle x-y, y-x\rangle-\langle x-y, x\rangle-\langle y, y\rangle \\
= & -\|g(x)-g(y)\|^{2}+\|g(x)\|^{2}-\langle g(y), g(x)\rangle+\|g(y)\|^{2} \\
\quad & +\|x-y\|^{2}-\|x\|^{2}+\langle y, x\rangle-\|y\|^{2} .
\end{aligned}
$$

If we move the terms $\langle g(y), g(x)\rangle$ and $\langle y, x\rangle$ on the right-hand side to the left-hand side of the above equality and divide both resulting sides by 2 , then we get:

$$
\begin{aligned}
& \langle g(x), g(y)\rangle-\langle x, y\rangle \\
& \quad=-\frac{1}{2}\|g(x)-g(y)\|^{2}+\frac{1}{2}\|x-y\|^{2}+\frac{1}{2}\|g(x)\|^{2}-\frac{1}{2}\|x\|^{2}+\frac{1}{2}\|g(y)\|^{2}-\frac{1}{2}\|y\|^{2} .
\end{aligned}
$$


Since $F$ is a real Hilbert space, it follows from (12)-(14) that:

$$
\begin{aligned}
&|\langle g(x), g(y)\rangle-\langle x, y\rangle| \\
& \leq \frac{1}{2}\left|\|g(x)-g(y)\|^{2}-\|x-y\|^{2}\right|+\frac{1}{2}\left|\|g(x)\|^{2}-\|x\|^{2}\right|+\frac{1}{2}\left|\|g(y)\|^{2}-\|y\|^{2}\right| \\
&= \frac{1}{2}|\|g(x)-g(y)\|+\|x-y\|||\|g(x)-g(y)\|-\|x-y\|| \\
& \quad+\frac{1}{2}|\|g(x)\|+\|x\|||\|g(x)\|-\|x\||+\frac{1}{2}|\|g(y)\|+\|y\|||\|g(y)\|-\|y\|| \\
& \leq \frac{1}{2}(2\|x-y\|+\varphi(x, y))|\|g(x)-g(y)\|-\|x-y\|| \\
&+\frac{1}{2}(2\|x\|+\varphi(x, 0))|\|g(x)\|-\|x\||+\frac{1}{2}(2\|y\|+\varphi(0, y))|\|g(y)\|-\|y\|| \\
& \leq \frac{1}{2}(2\|x-y\|+\varphi(x, y)) \varphi(x, y)+\frac{1}{2}(2\|x\|+\varphi(x, 0)) \varphi(x, 0) \\
&+\frac{1}{2}(2\|y\|+\varphi(0, y)) \varphi(0, y) \\
&=\|x-y\| \varphi(x, y)+\|x\| \varphi(x, 0)+\|y\| \varphi(0, y)+\frac{1}{2} \varphi(x, y)^{2}+\frac{1}{2} \varphi(x, 0)^{2}+\frac{1}{2} \varphi(0, y)^{2} \\
&= \psi(x, y)
\end{aligned}
$$

for all $x, y \in D_{0}$.

Then it follows from the first two conditions for $\varphi$ that $\psi$ satisfies the condition (8) for any $x, y \in D_{0}$. Applying Theorem 5 with $g$ and $\psi$ instead of $f$ and $\varphi$, we can complete the rest of the proof.

When we set $\varphi(x, y)=0$ for $x=0$ or $y=0$, Theorem 6 will be considerably simplified. The control function $\varphi(x, y)=\theta\|x\|^{p}\|y\|^{q}$ was first used by Rassias [33] for approximate additive functions. The following corollary is an example of applying Theorem 6 .

Corollary 1. Let $E$ and $F$ be Hilbert spaces over $\mathbb{R}$. Assume that $d, p$, $q$, and $\theta$ are constants with $d \geq 0, p \geq 1, q \geq 1$, and $\theta>0$. Moreover, let $D=\{x \in E:\|x\| \leq d\}$. If a function $f: E \rightarrow F$ satisfies the following inequality:

$$
|\|f(x)-f(y)\|-\|x-y\|| \leq \theta\|x\|^{p}\|y\|^{q}
$$

for all $x, y \in D$, then there exists a unique linear isometry $U: E \rightarrow F$ such that:

$$
\|f(x)-U(x)-f(0)\| \leq \begin{cases}\frac{\sqrt{2}}{2} \theta\|x\|^{p+q} & (\text { for } p>1 \text { and } q>1), \\ \sqrt{\frac{1}{2} \theta^{2}\|x\|^{2 q+2}+\theta\|x\|^{q+2}} & (\text { for } p=1 \text { and } q>1) \\ \sqrt{\frac{1}{2} \theta^{2}\|x\|^{2 p+2}+\theta\|x\|^{p+2}} & (\text { for } p>1 \text { and } q=1) \\ \sqrt{\frac{1}{2} \theta^{2}\|x\|^{4}+2 \theta\|x\|^{3}} & (\text { for } p=q=1)\end{cases}
$$

for all $x \in D$.

Proof. Considering the condition (6), we select a constant $c$ satisfying $c>1$ and we define $\varphi(x, y)=\theta\|x\|^{p}\|y\|^{q}$ for any $x, y \in E$. Then, we easily verify that:

$$
\lim _{m, n \rightarrow \infty} c^{m+n}\left\|\frac{1}{c^{m}} x-\frac{1}{c^{n}} y\right\| \varphi\left(\frac{1}{c^{m}} x, \frac{1}{c^{n}} y\right)=\lim _{m, n \rightarrow \infty} \theta \frac{c^{m+n}}{c^{p m+q n}}\left\|\frac{1}{c^{m}} x-\frac{1}{c^{n}} y\right\|\|x\|^{p}\|y\|^{q}=0
$$


for all $x, y \in D$. Moreover, we have:

$$
\lim _{m, n \rightarrow \infty} c^{m+n} \varphi\left(\frac{1}{c^{m}} x, \frac{1}{c^{n}} y\right)^{2}=\lim _{m, n \rightarrow \infty} \theta^{2} \frac{c^{m+n}}{c^{2 p m+2 q n}}\|x\|^{2 p}\|y\|^{2 q}=0
$$

for all $x, y \in D$. Further, with the definition of $\psi$ given in Theorem 6, we get:

$$
\lim _{n \rightarrow \infty} c^{n} \psi\left(\frac{1}{c^{n}} x, x\right)= \begin{cases}0 & (\text { for } p>1) \\ \theta\|x\|^{q+2} & (\text { for } p=1)\end{cases}
$$

and

$$
\lim _{n \rightarrow \infty} c^{n} \psi\left(x, \frac{1}{c^{n}} x\right)= \begin{cases}0 & (\text { for } q>1) \\ \theta\|x\|^{p+2} & (\text { for } q=1)\end{cases}
$$

for all $x \in D$. Thus, according to Theorem 6, there exists a unique linear isometry $U: E \rightarrow F$ satisfying the given inequality for all $x \in D$.

Since $\|f(x)-f(y)\| \geq 0$ and $\|x-y\| \geq 0$ for any $x, y \in E$, it holds that for all $x, y \in E$,

$$
\begin{aligned}
& \|f(x)-f(y)\|^{2}=\|x-y\|^{2} \\
& \quad \Longleftrightarrow\|f(x)-f(y)\|=\|x-y\| \text { or }\|f(x)-f(y)\|=-\|x-y\| \\
& \quad \Longleftrightarrow\|f(x)-f(y)\|=\|x-y\| \text { or } x=y \\
& \quad \Longleftrightarrow\|f(x)-f(y)\|=\|x-y\| .
\end{aligned}
$$

Hence, the set of solutions to the functional equation $\|f(x)-f(y)\|=\|x-y\|$ is the same as the set of solutions to the equation $\|f(x)-f(y)\|^{2}=\|x-y\|^{2}$, but in both cases stability results are different, as can be seen in Theorems 6 and 7:

Theorem 7. Let $E$ and $F$ be Hilbert spaces over $\mathbb{R}$. Assume that a function $\varphi: E \times E \rightarrow[0, \infty)$ satisfies the condition (8) for all $x, y \in D_{0}$. If a function $f: E \rightarrow F$ satisfies the inequality:

$$
\left|\|f(x)-f(y)\|^{2}-\|x-y\|^{2}\right| \leq \varphi(x, y)
$$

for all $x, y \in D$, then there exists a unique linear isometry $U: E \rightarrow F$ such that:

$$
\|f(x)-U(x)-f(0)\| \leq \sqrt{\psi(x, x)+\lim _{n \rightarrow \infty} c^{n} \psi\left(\frac{1}{c^{n}} x, x\right)+\lim _{n \rightarrow \infty} c^{n} \psi\left(x, \frac{1}{c^{n}} x\right)}
$$

for all $x \in D$, where $\psi(x, y)=\frac{1}{2} \varphi(x, y)+\frac{1}{2} \varphi(x, 0)+\frac{1}{2} \varphi(0, y)$ for all $x, y \in E$.

Proof. If we put $g(x)=f(x)-f(0)$ in (15), then $g$ also satisfies the inequality (15) for all $x, y \in D$. By (14) and (15) with $g$ in place of $f$, we have:

$$
\begin{aligned}
& |\langle g(x), g(y)\rangle-\langle x, y\rangle| \\
& \quad \leq \frac{1}{2}\left|\|g(x)-g(y)\|^{2}-\|x-y\|^{2}\right|+\frac{1}{2}\left|\|g(x)\|^{2}-\|x\|^{2}\right|+\frac{1}{2}\left|\|g(y)\|^{2}-\|y\|^{2}\right| \\
& \quad \leq \frac{1}{2} \varphi(x, y)+\frac{1}{2} \varphi(x, 0)+\frac{1}{2} \varphi(0, y) \\
& \quad=\psi(x, y)
\end{aligned}
$$

for any $x, y \in D$. Moreover, the $\psi$ satisfies the condition (8) for all $x, y \in D$. According to Theorem 5 , there exists a unique linear isometry $U: E \rightarrow F$ satisfying the given inequality for all $x \in D$. 
Finally, the following corollary can be demonstrated by using Theorem 7 .

Corollary 2. Let $E$ and $F$ be Hilbert spaces over $\mathbb{R}$. Assume that $d$, $p$, and $\varepsilon$ are constants with $d \geq 0,0<p \leq 1$, and $\varepsilon>0$. Moreover, let $D=\{x \in E:\|x\| \geq d\}$. If a function $f: E \rightarrow F$ satisfies the inequality:

$$
\left|\|f(x)-f(y)\|^{2}-\|x-y\|^{2}\right| \leq \varepsilon\|x-y\|^{p}
$$

for all $x, y \in D_{0}$, then there exists a unique linear isometry $U: E \rightarrow F$ such that:

$$
\|f(x)-U(x)-f(0)\| \leq \begin{cases}\sqrt{\varepsilon\|x\|^{p}} & (\text { for } 0<p<1), \\ \sqrt{3 \varepsilon\|x\|} & (\text { for } p=1)\end{cases}
$$

for all $x \in D_{0}$.

Proof. Considering the condition (6), we may select a constant $c$ satisfying $0<c<1$. If we define $\varphi(x, y)=\varepsilon\|x-y\|^{p}$ for all $x, y \in E$, then we easily show that:

$$
\lim _{m, n \rightarrow \infty} c^{m+n} \varphi\left(\frac{1}{c^{m}} x, \frac{1}{c^{n}} y\right)=\lim _{m, n \rightarrow \infty} \varepsilon c^{(1-p)(m+n)}\left\|c^{n} x-c^{m} y\right\|^{p}=0
$$

for all $x, y \in D_{0}$. Moreover, with the definition of $\psi$ given in Theorem 7, we obtain:

$$
\lim _{n \rightarrow \infty} c^{n} \psi\left(\frac{1}{c^{n}} x, x\right)= \begin{cases}0 & (\text { for } 0<p<1), \\ \varepsilon\|x\| & (\text { for } p=1)\end{cases}
$$

and

$$
\lim _{n \rightarrow \infty} c^{n} \psi\left(x, \frac{1}{c^{n}} x\right)= \begin{cases}0 & (\text { for } 0<p<1), \\ \varepsilon\|x\| & (\text { for } p=1)\end{cases}
$$

for all $x \in D_{0}$. Finally, according to Theorem 7 , there exists a unique linear isometry $U: E \rightarrow F$ such that the given inequality holds for all $x \in D$. Obviously, this inequality holds also for $x=0$.

We may compare Corollary 2 with Theorem 5 of [32], in which the stability of the Equation (1) was proved under the assumptions that $D=\{x \in E:\|x\| \leq d\}$ and $p>1$.

\section{Discussion}

This paper focused on stability in the restricted domain of isometries. Most other papers address the stability problem of isometries defined in the whole space. This is the difference between this paper and most other papers.

Fickett's Thereom (Theorem 1) provided an important motivation for writing this paper: The upper bound associated with inequality in Fickett's theorem becomes very large for any sufficiently small $\varepsilon$ in comparison to $\varepsilon$. This is a big drawback of Fickett's theorem. Thus, the work of further improving Fickett's theorem has to be attractive. Although this paper did not achieve that goal completely, we wrote this paper as part of an attempt to further improve the theorem of Fickett.

Author Contributions: Conceptualization, S.-M.J.; methodology, G.C. and S.-M.J.; validation, G.C. and S.-M.J.; investigation, G.C. and S.-M.J.; writing-original draft preparation, G.C. and S.-M.J.; writing-review and editing, G.C. and S.-M.J.; project administration, S.-M.J.; funding acquisition, G.C. and S.-M.J. All authors have read and agreed to the published version of the manuscript.

Funding: This work was supported by the National Research Foundation of Korea (NRF) grant funded by the Korea government (MSIT) (No. 2020R1F1A1A01049560). This work was supported by 2020 Hongik University Research Fund. 
Institutional Review Board Statement: Not applicable.

Informed Consent Statement: Not applicable.

Data Availability Statement: Not applicable.

Conflicts of Interest: The authors declare no conflict of interest.

\section{References}

1. Hyers, D.H.; Ulam, S.M. On approximate isometries. Bull. Am. Math. Soc. 1945, 51, 288-292. [CrossRef]

2. Bourgin, D.G. Approximate isometries. Bull. Am. Math. Soc. 1946, 52, 704-714. [CrossRef]

3. Hyers, D.H.; Ulam, S.M. Approximate isometries of the space of continuous functions. Ann. Math. 1947, 48, 285-289. [CrossRef]

4. Bourgin, D.G. Approximately isometric and multiplicative transformations on continuous function rings. Duke Math. J. 1949, 16, 385-397. [CrossRef]

5. Bourgin, R.D. Approximate isometries on finite dimensional Banach spaces. Trans. Am. Math. Soc. 1975, 207, 309-328. [CrossRef]

6. Gruber, P.M. Stability of isometries. Trans. Am. Math. Soc. 1978, 245, 263-277. [CrossRef]

7. Gevirtz, J. Stability of isometries on Banach spaces. Proc. Am. Math. Soc. 1983, 89, 633-636. [CrossRef]

8. Omladič, M.; Šemrl, P. On non linear perturbations of isometries. Math. Ann. 1995, 303, 617-628. [CrossRef]

9. Dolinar, G. Generalized stability of isometries. J. Math. Anal. Appl. 2000, 242, 39-56. [CrossRef]

10. Šemrl, P. Hyers-Ulam stability of isometries on Banach spaces. Aequ. Math. 1999, 58, 157-162. [CrossRef]

11. Baker, J.A. Isometries in normed spaces. Amer. Math. Mon. 1971, 78, 655-658. [CrossRef]

12. Bhatia, R.; Šemrl, P. Approximate isometries on Euclidean spaces. Amer. Math. Mon. 1997, 104, 497-504. [CrossRef]

13. Chmieliński, J. On the superstability of the generalized orthogonality equation in Euclidean spaces. Ann. Math. Sil. 1994, 8, 127-140.

14. Chmieliński, J. Stability of the orthogonality preserving property in finite-dimensional inner product spaces. J. Math. Anal. Appl. 2006, 318, 433-443. [CrossRef]

15. Fickett, J.W. Approximate isometries on bounded sets with an application to measure theory. Stud. Math. 1981, 72, 37-46. [CrossRef]

16. Jung, S.-M. Hyers-Ulam-Rassias stability of isometries on restricted domains. Nonlinear Stud. 2001, 8, 125-134.

17. Jung, S.-M. Asymptotic properties of isometries. J. Math. Anal. Appl. 2002, 276, 642-653. [CrossRef]

18. Lindenstrauss, J.; Szankowski, A. Non linear perturbations of isometries. Astérisque 1985, 131, 357-371.

19. Rassias, T.M. Properties of isometric mappings. J. Math. Anal. Appl. 1999, 235, 108-121. [CrossRef]

20. Rassias, T.M. Isometries and approximate isometries. Internat. J. Math. Math. Sci. 2001, 25, 73-91. [CrossRef]

21. Rassias, T.M.; Sharma, C.S. Properties of isometries. J. Natural Geom. 1993, 3, 1-38.

22. Skof, F. Sulle $\delta$-isometrie negli spazi normati. Rend. Mat. Ser. VII Roma 1990, 10, 853-866.

23. Skof, F. On asymptotically isometric operators in normed spaces. Lombardo Acad. Sci. Lett. Rend. A 1997, 131, 117-129.

24. Swain, R.L. Approximate isometries in bounded spaces. Proc. Am. Math. Soc. 1951, 2, 727-729. [CrossRef]

25. Väisälä, J. Isometric approximation property of unbounded sets. Result. Math. 2003, 43, 359-372. [CrossRef]

26. Chmielinski, J.; Jung, S.-M. The stability of the Wigner equation on a restricted domain. J. Math. Anal. Appl. 2001, 254, 309-320. [CrossRef]

27. Chmieliński, J. On the Hyers-Ulam stability of the generalized orthogonality equation in real Hilbert spaces. In Stability of Mappings of Hyers-Ulam Type; Rassias, T.M., Tabor, J., Eds.; Hadronic Press: Palm Harbor, FL, USA, 1994; pp. 31-41.

28. Chmieliński, J. The stability of the Wigner equation in complex Hilbert spaces. Wyż. Szkoła Ped. Kraków Rocznik Nauk.-Dydakt. Prace Matematyczne 1998, 15, 49-55.

29. Chmieliński, J. On a singular case in the Hyers-Ulam-Rassias stability of the Wigner equation. J. Math. Anal. Appl. 2004, 289, 571-583. [CrossRef]

30. Wigner, E.P. Gruppentheorie und ihre Anwendungen auf die Quantenmechanik der Atomspektren; Vieweg und Sohn: Braunschweig, Germany, 1931.

31. Hyers, D.H. On the stability of the linear functional equation. Proc. Natl. Acad. Sci. USA 1941, 27, 222-224. [CrossRef]

32. Jung, S.-M.; Kim, B. Stability of isometries on restricted domains. J. Korean Math. Soc. 2000, 37, 125-137.

33. Rassias, J.M. On a new approximation of approximately linear mappings by linear mappings. Discuss. Math. 1985, 7, 193-196. 